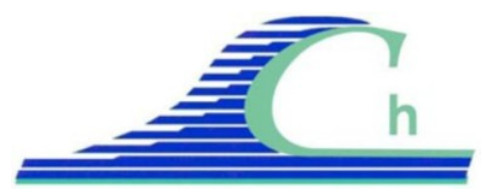

XII ${ }^{\text {èmes }}$ Journées Nationales Génie Côtier - Génie Civil

Cherbourg, 12-14 juin 2012

DOI:10.5150/jngcgc.2012.014-T @ Editions Paralia CFL

disponible en ligne - http://www.paralia.fr - available online

\title{
Stratégie de modélisation hydrodynamique pour évaluer les rejets thermiques en milieu marin
}

\author{
Guillaume TABURET ${ }^{1}$, Charles TALBOT ${ }^{1}$, Philippe CRANEGUY ${ }^{1}$, \\ Jean ROUSSELET ${ }^{2}$, Antoine MANGIN ${ }^{1}$
}

1. ACRI-ST, 260 route du Pin Montard, BP 234, 06904 Sophia Antipolis Cedex, France.

2. DALKIA centre PACA, Le Laser, 184 Cours Lafayette, 69441 Lyon cedex 03,

France.

Guillaume.Taburet@acri-st.fr

\begin{abstract}
Résumé :
L'objectif du projet OPTIMA-PAC est de développer et de mettre en place un service d'aide à la décision pour l'implantation et le fonctionnement des pompes à chaleur eau de mer (PAC). Ce service dédié aux besoins des industriels et des collectivités territoriales, intègre les enjeux environnementaux et d'optimisation énergétique.

On donne ici un aperçu de la stratégie de modélisation numérique des conditions météoocéaniques retenue dans le cadre du projet OPTIMA-PAC. Les résultats de ces simulations numériques sont exploités aussi bien pour la préparation des campagnes de mesures en mer (prévisions opérationnelles à haute résolution des conditions météoocéaniques) que pour l'évaluation de l'impact environnemental et l'optimisation énergétique des PAC (rejeux des conditions météo-océanique).
\end{abstract}

\section{Mots-clés :}

Modélisation - Energies marines renouvelables - Pompe à chaleur - Optimisation énergétique - Milieu marin côtier - Impact environnemental

\section{Introduction}

Dans un monde de plus en plus énergivore où les ressources fossiles s'amenuisent, il est nécessaire de trouver des solutions locales de production adaptées au monde de demain. Les mers et les océans représentent un réservoir énergétique naturel qui pourrait assurer une partie des besoins énergétiques des franges littorales, dans le cadre de projets d'aménagements urbain touchant des constructions neuves ou des réhabilitations.

La pompe à chaleur (PAC) eau de mer répond à ces nouveaux enjeux. Elle repose sur un système thermodynamique qui permet de transférer de la chaleur à partir d'une source froide ou chaude (eau de mer) via un réseau de chaleur chaud ou froid jusqu'à un utilisateur final. Grâce à un excellent rendement énergétique cette solution permet, sur un même bâtiment de réduire les consommations énergétiques de plus de 30\% et les émissions de gaz à effet de serre de plus de 60\% par rapport à une solution traditionnelle chaudière et groupe-froid. Au contraire une gestion mal maitrisée de ce gisement pourrait conduire à une remise en cause de cette écotechnologie. 
Les énergies marines restent encore assez peu développées en zone littorale. La PAC eau de mer est pourtant une technologie mature offrant des applications concrètes et innovantes de valorisation de cette énergie renouvelable. De tels systèmes existent déjà sur le littoral méditerranéen et depuis près de 30 ans sur le territoire monégasque. DALKIA, leader du projet OPTIMA-PAC, exploite actuellement à Monaco trois installations majeures de type PAC eau de mer.

Le projet OPTIMA-PAC, labellisé par le pôle Mer PACA, est soutenu par l'Etat français, la principauté de Monaco et la Communauté Urbaine Marseille Provence (CUMP), le Conseil Général 06 (CG06) et le Conseil Régional PACA (CR PACA). Ce projet s'appuie sur les retours d'expériences des installations de Monaco et repose sur des sites pilote en territoire monégasque. Porté par DALKIA, ce projet, qui a débuté en 2011, regroupe des acteurs clés tant dans le secteur industriel (VEOLIA, BARRIQUAND, CRUDELLI, ACRI-ST, ACRI-IN) que dans celui de la recherche (ECOMERS, UPMC/CNRS(OOV)).

Le projet OPTIMA-PAC consiste à anticiper le développement du marché soutenu par les orientations du Grenelle de l'Environnement et de la Mer et à préparer les conditions durables de développement de la filière industrielle des PAC eau de mer. Ce projet vise à mettre au point des outils d'aide à la décision permettant aux aménageurs de mieux appréhender les futurs projets de PAC, notamment à l'aide d'outils prévisionnels faisant appel au couplage de modèles et de bio-indicateurs. Pour les bureaux d'études et les exploitants, ce projet permet de définir une conception et gestion optimisées des ouvrages de captage et relargage en mer de l'eau pompée, en vue d'une optimisation énergétique et environnementale des PAC. Enfin ce projet permet aux scientifiques de mettre au point des indicateurs de suivi de milieux servant aux études d'impact de futurs projets de PAC eau de mer.

La phase de modélisation présentée dans cet article ne constitue qu'une partie de l'apport en données environnementales et sur lesquels s'ajoutent les autres tâches du projet. Dans un premier temps, nous présenterons la stratégie de modélisation océanique et météorologique en précisant des perspectives exploratoires envisagées pour en améliorer les performances. Enfin nous aborderons la définition des scénarios de validation de diffusion thermique puis d'optimisation de prises d'eau et de rejet.

\section{Modélisation océanique/météorologique et validation}

\subsection{Modélisation hydrodynamique}

ACRI-ST a mis en place et opère une chaîne de calcul de prévision océanographique sur le littoral Sud-Est de la France, depuis l'ouest de Marseille jusqu’à Monaco. La stratégie d'emboîtement de modèles a été mise à profit avec succès, en utilisant comme données à l'initialisation et aux limites celles issues de l'emprise MENOR du code MARS3D (résolution $1.2 \mathrm{~km}$ ), opérée par l'IFREMER. Le domaine régional, nommé PACA et de 


\section{XII ${ }^{\text {èmes }}$ Journées Nationales Génie Côtier - Génie Civil \\ Cherbourg, 12-14 juin 2012}

résolution $400 \mathrm{~m}$, fournit en temps réel des prévisions à 48 heures des conditions hydrodynamiques (courant, température, salinité) depuis 2007 (voir figure 1). Les résultats de ce système opérationnel de prévision ont été calibrés pour représenter de manière réaliste les courants et l'hydrologie dans la zone côtière. Ils sont exploités notamment pour prédire en temps réel l'impact sanitaire de rejets urbains dans la zone d'Antibes au sein du projet GIRAC (gestion des impacts des rejets d'assainissement côtiers piloté par Veolia Eau).

Dans le cadre du projet OPTIMA-PAC, une stratégie de modèles gigognes a été envisagée. Deux modèles fines échelles, de résolution respective 150 et $50 \mathrm{~m}$, ont été imbriqués dans le modèle PACA (voir figure 2). De telle résolution spatiale sont en effet indispensable pour représenter les phénomènes de petite échelle et de fait mieux évaluer l'impact environnemental des PAC.

Le modèle hydrodynamique MARS3D (LAZURE \& DUMAS, 2007) développé par IFREMER est un algorithme de calcul des courants marins, des hauteurs d'eau, du champ de densité et des concentrations en éléments solubles (ou en suspension temporaire) transportés par les courants, dans les 3 dimensions spatiales. Il utilise un schéma aux différences finies sur un maillage régulier en longitude/latitude sur l'horizontale et en coordonnées sigma sur la verticale, permettant de reproduire fidèlement les fonds marins qui peuvent être absolument quelconques et comporter, par exemple, îles, hauts fonds, bancs découvrants et estrans. Le cœur hydrodynamique du modèle résout les équations primitives d'un milieu incompressible, hydrostatique suivant l'hypothèse de Boussinesq, et s'appuie sur des schémas classiques en différences finies sur une grille Arakawa $C$ (verticale et horizontale). Le modèle MARS exploite la technique d'emboîtement de modèles sous forme gigogne. Par cette technique un modèle de grande emprise et de moindre résolution contient lui-même un ou plusieurs sous modèles (loupes, cf. figure 2).

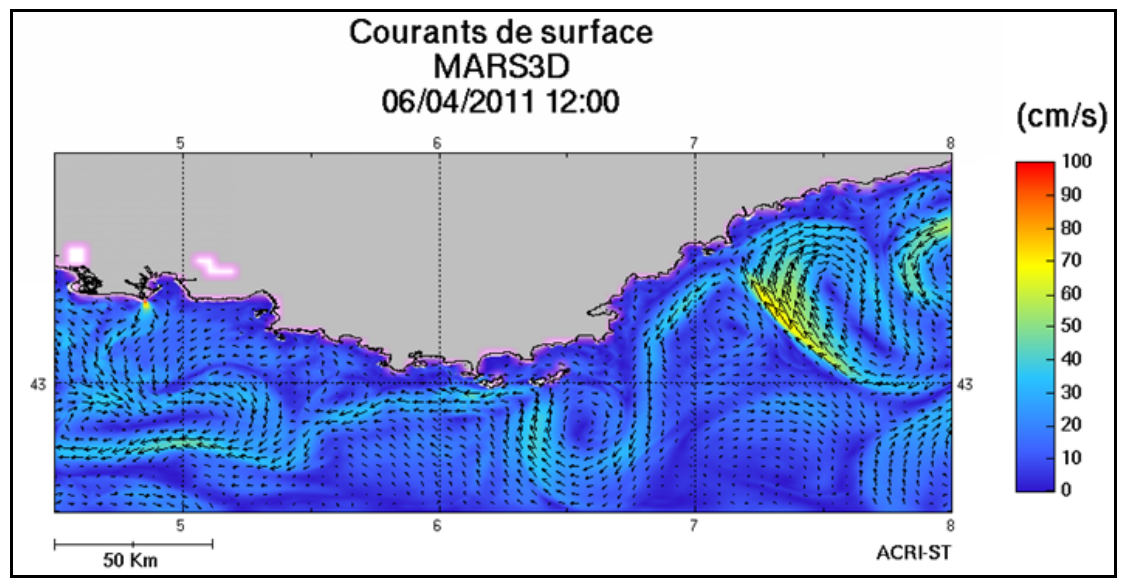

Figure 1. Prévision du courant de surface à 12 h00 le 06/04/2011 simulée par l'emprise PACA du système opérationnel de prévision d'ACRI-ST. 


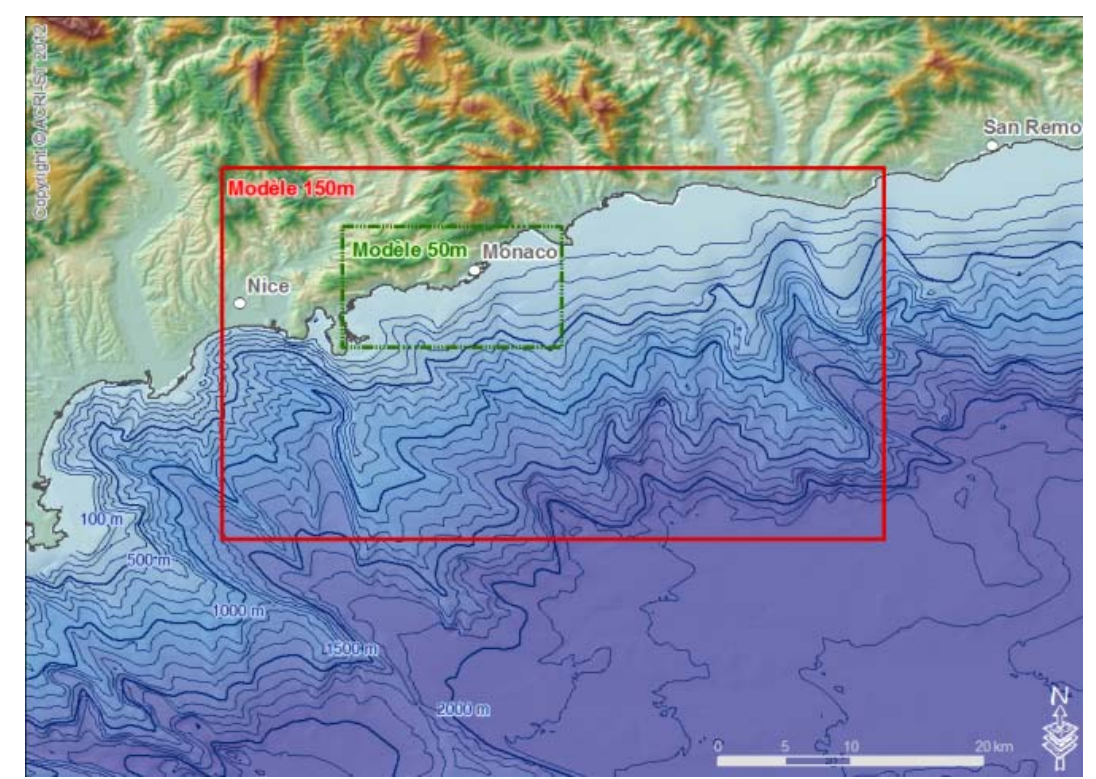

Figure 2. Modèles hautes résolutions centrés autour de la principauté de Monaco et emboités dans l'emprise PACA.

\subsection{Modélisation météorologique}

Le forçage par le vent est un élément essentiel de l'hydrodynamique de la Baie monégasque. Outre la nécessité de disposer de données de vent réalistes, il est essentiel d'assurer la continuité et de la cohérence des forçages météorologiques qui seront exploités pour simuler l'hydrodynamique de la zone d'étude.

ACRI-ST a mis en place et opère depuis 2006, quotidiennement, un système opérationnel de prévision météorologique sur la Méditerranée Nord Occidentale. Ce système est composé de 3 modèles imbriqués, du modèle atmosphèrique MM5 (Le modèle MM5 est un modèle tridimensionnel non hydrostatique en coordonnées verticales qui s’attache à modéliser les phénomènes météorologiques à une échelle régionale, dont la résolution spatiale peut varier entre $200 \mathrm{~m}$ et $100 \mathrm{~km}$. Ce modèle est un modèle conçu par la Pennsylvania State University (PSU) et le National Center for Atmospheric Research (NCAR) pour la prévision et la simulation de la circulation atmosphérique) (DUDHIA et al., 2005) et de résolution respective d'environ $27 \mathrm{~km}$ (domaine Europe), $9 \mathrm{~km}$ (domaine Lion) et $3 \mathrm{~km}$ (domaine Paca), dont les emprises sont présentées dans la figure 3 et qui sont alimentés par les prévisions assimilées du Centre National de Prévision Environnemental américain (NCEP).

Les résultats d'analyse du modèle à haute résolution (domaine Paca), conservés dans notre base de données en interne, sont extraits et exploités pour la présente étude.

Les résultats du système opérationnel de prévision météorologique d'ACRI-ST sont exploités depuis de nombreuses années par la communauté scientifique. Ainsi, le modèle opérationnel MARS3D de prévision de la circulation océanique en Méditerranée Nord Occidentale (modèle MENOR) de l'IFREMER est forcé par les champs de surface 


\section{XII ${ }^{\text {èmes }}$ Journées Nationales Génie Côtier - Génie Civil \\ Cherbourg, 12-14 juin 2012}

du système opérationnel d'ACRI-ST. Par ailleurs, nombres d'articles scientifiques et de thèses portant sur la modélisation à haute-résolution de la circulation aux abords du Golfe du Lion utilisent comme forçages météorologiques ceux issus de nos chaînes opérationnelles (par exemple : SCHAEFFER et al., 2011). Ces travaux ont notamment permis de démontrer la bonne qualité des prévisions de vent issues de notre système de prévision, notamment à très haute résolution (voir figure 4).

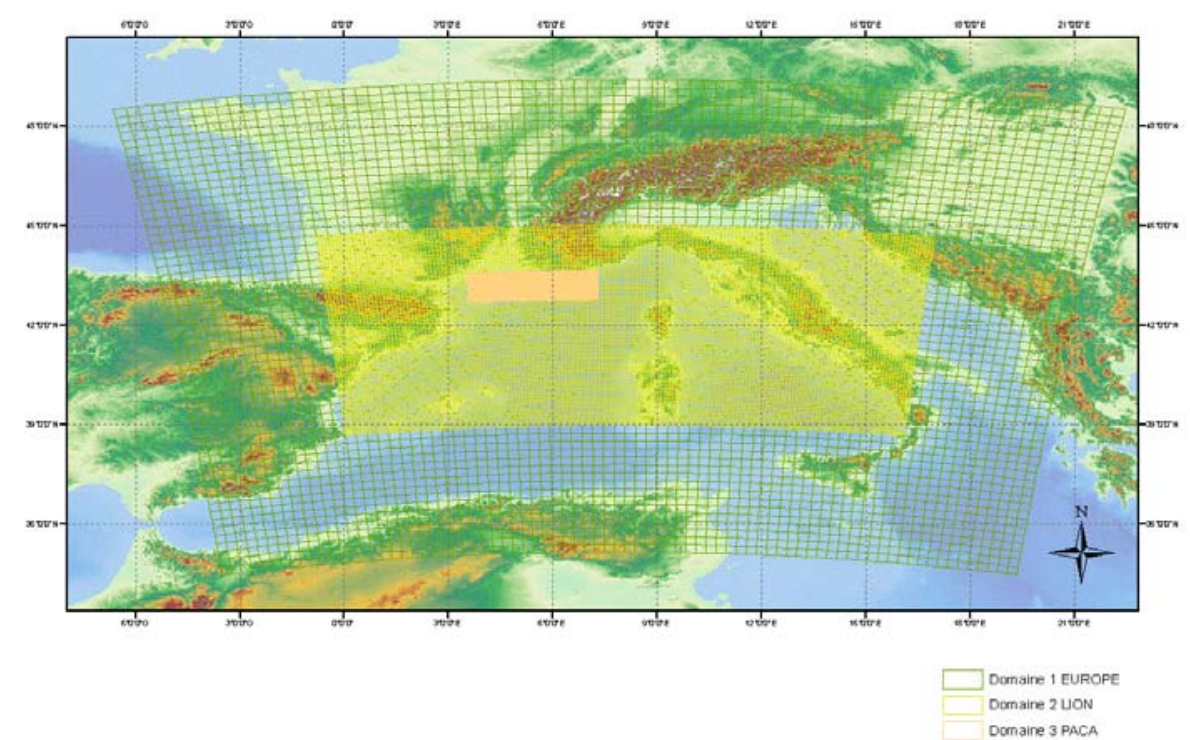

Figure 3. Emprises du système opérationnel météorologique mis en place et exploité quotidiennement par ACRI-ST. La résolution horizontale du modèle sur le domaine Europe est d'environ 27 km, sur le domaine Lion est d'environ 9 km, et sur le domaine Paca est d'environ $3 \mathrm{~km}$.

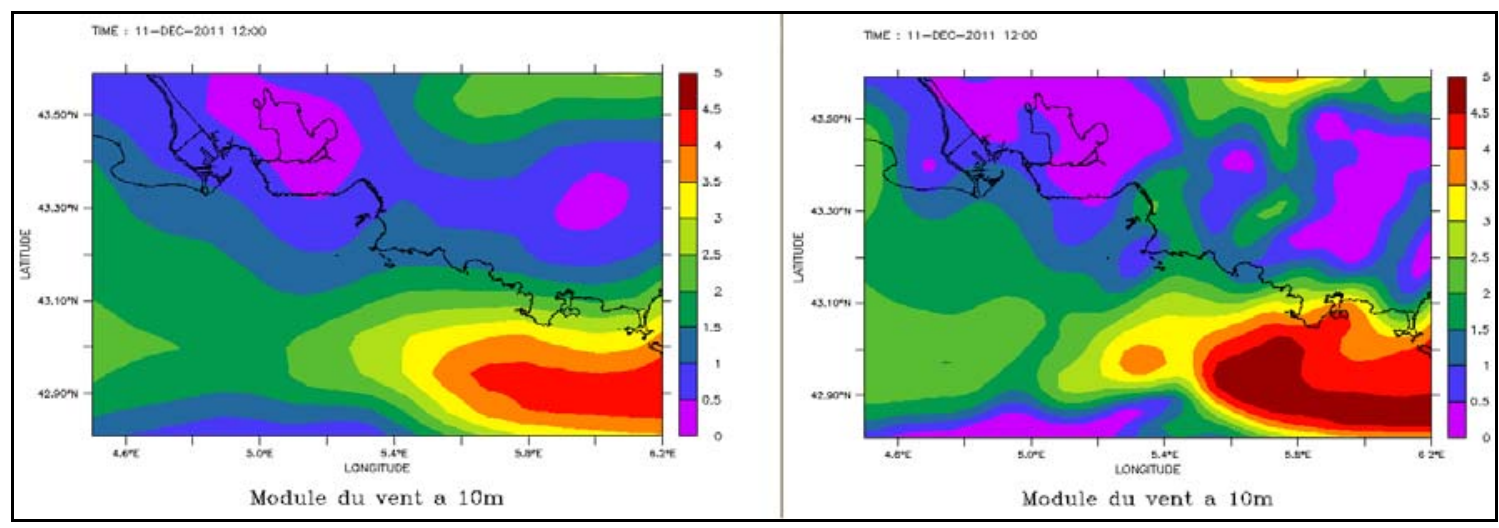

Figure 4. Illustration de l'apport de la haute résolution spatiale pour la simulation de l'intensité du vent : simulation à $9 \mathrm{~km}$ de résolution (figure de gauche), simulation à $3 \mathrm{~km}$ de résolution (figure de droite). 
En parallèle de cette configuration MM5, ACRI-ST met en place sur Monaco une configuration à très haute résolution, descendant jusqu'à la centaine de mètre, à l'aide du modèle météorologique WRF (Weather Reseach and Forecasting). L'objectif est à terme de mieux représenter les processus de petite échelle sur Monaco pour forcer le modèle marin à l'aide de données météorologiques les plus fine possible.

Le modèle WRF est un modèle multi-échelle de nouvelle génération conçu par le National Center of Atmospheric Research (NCAR), aux Etats-Unis, pour prévoir et modéliser la circulation atmosphérique. Il est conçu à la fois pour des prévisions opérationnelles et les besoins de la recherche atmosphérique. Son architecture de modèle plus flexible en fait le successeur du modèle MM5 pour la communauté scientifique.

Les conditions initiales et aux limites viennent des données du modèle NCEP- GFS à $0.5^{\circ}$ de résolution. Le système opérationnel à ACRI-ST avec le modèle WRF fournit actuellement 72 heures de prévision par pas de temps de 1 heure. Les prévisions incluent entre autre la pression atmosphérique, la vitesse et la direction du vent, la température, la couverture nuageuse totale et les précipitations.

Dans le cadre du projet européen FP7-Environnement PASODOBLE, ACRI-ST exploite cette chaîne sur une région assez large et couvrant, à de multiples résolutions (18km, 6 km et 2 km), la partie française de la mer Méditerranée.

\subsection{Calibration, validation des modèles marins.}

\section{- Calibration :}

L'étape de calibration exploitera l'ensemble des données environnementales, collectées par les intervenants du projet lors des diverses campagnes de mesure, et/ou leur synthèse statistique. Elle pointera essentiellement sur les variables d'intérêt pour le projet : courant, température et structure thermique verticale.

A partir du système décrit, des périodes longues seront simulées ( 1an).De ces premiers résultats, nous vérifierons la cohérence avec les mêmes résultats issus des modèles de forçages d'emprise supérieures (en particulier modèle PACA) ;

La similitude statistique entre nos simulations et les conclusions consignées et obtenues lors des diverses campagnes en mer.

En pratique les évolutions temporelles et les roses de courant simulées seront comparées avec celles issues des observations.

\section{- Validation :}

Dans le cadre de la présente étude, le modèle d'application sera exploité pour évaluer les rejets thermiques au cours de situations météo-océaniques type, représentatives d'une année, situations qui seront définies à partir de l'analyse statistique des mesures. Une méthode d'analyse statistique des écarts modèle à ces situations ou méthode Novelty (MARKOS \& SAMEER, 2003) sera mise en place. Cette méthode, issue de l'analyse discriminante des clusters, permet de classifier une situation au sein 


\section{XII ${ }^{\text {èmes }}$ Journées Nationales Génie Côtier - Génie Civil \\ Cherbourg, 12-14 juin 2012}

de situations type préétablies. Les situations type correctement et non correctement reproduites par le modèle seront identifiées. Il s'agira de distinguer les phénomènes parfaitement reproduits, de ceux pour lesquels une incertitude non négligeable oblige à nuancer l'utilisation (par exemple: capacité du modèle à reproduire la durée, l'extension, l'intensité d'upwelling ou la variabilité des courants).

- Méthode semi-pronostique :

Si les échelles régionales ne sont pas suffisamment bien reproduites par les modèles gigognes nous envisageons de mettre en place la méthode semi-pronostique (GREATBATCH et al., 2004). Cette méthode peut être utilisée dans le cadre des modèles emboitées pour transférer de l'information des modèles grandes échelles aux modèles fines échelles. Cette méthode repose sur une discrétisation de la densité instantanée en une combinaison linéaire de la densité modèle et d'une densité d'entrée (pouvant être celle d'un modèle d'échelle différente). Ce travail est encore à l'étude et fait l'objet de recherche afin d'en parfaire l'utilisation.

\section{Perspectives}

Du fait de son principe thermodynamique, le procédé de pompe à chaleur peut modifier l'environnement marin proche par la création d'un gradient thermique entre les eaux pompées et les eaux rejetées. Cet impact qui peut éventuellement influencer le fonctionnement des écosystèmes côtiers, va dépendre des conditions locales et des caractéristiques de l'installation. En parallèle, l'intensification du gradient thermique entre les eaux pompées et les eaux rejetées, peut affecter le rendement des pompes à chaleur eau de mer.

Les scénarios mis en place porteront donc essentiellement sur les positions respectives des captages et des rejets et sur la possible mutualisation des captages (par exemple à plus grande profondeur sous la thermocline, ou par un aqueduc commun, ...). Nous tiendrons compte de diverses considération émanant des exploitants de pompes à chaleur et notamment des données de fonctionnement optimum suivant la température de captage de l'eau de mer et ses possibles variations.

\section{Références bibliographiques}

DUDHIA J., GILL D., MANNING K., WANG W., BRUYERE C. (2005). PSU/NCAR Mesoscale Modeling System Tutorial Class Notes and Users' Guide (MM5 Modeling). GREATBATCH R.J., SHENG J., EDEN C., TANG L., ZHAI X., ZHAO J. (2004). The semi-prognostic method. Continental Shelf Research, Volume 24, pp 2149-2165. doi:10.1016/j.csr.2004.07.009

LAZURE P., DUMAS F. (2007). An external-internal mode coupling for a 3D hydrodynamical model for applications at regional scale (MARS). Advances in Water Resources, Volume 31, pp 233-250. doi:10.1016/j.advwatres.2007.06.010 
Thème 1 - Hydrodynamique côtière

MARKOS M., SAMEER S. (2003). Novelty Detection: A Review Part 1: Statistical Approaches. Signal Processing, Volume 83, Issue 12, pp 2481-2497.

SCHAEFFER A., MOLCARD A., FORGET P., FRAUNIÉ P., GARREAU P. (2011).

Generation mechanism of mesoscale eddy in the Gulf of Lions: radar observation and modelling. Ocean Dynamics, Volume 61, pp 1587-1609. doi:10.1007/s10236-011-0482-8 